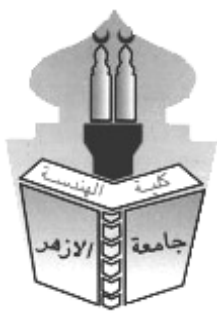

\title{
BUILDING SUSTAINABILITY: TOWARDS A CONTEMPORARY STRATEGY FOR SUSTAINABLE DEVELOPMENT
}

\author{
*Rania Said Sayd Mourad ${ }^{1}$, Mohamed Zakria El-Dars ${ }^{2}$ and Alaa-aldien Alsaied Farid ${ }^{2}$ \\ Architect, Development, Research \& Studies, Department Fund, Ministry of Housing, \\ Cairo, Egypt. \\ ${ }^{2}$ Department of Architecture, Faculty of Engineering, Al-Azhar University, Cairo, Egypt. \\ *Corresponding author E-mail: architecture_rania@hotmail.com,
}

\begin{abstract}
The study examined the concept of sustainability at the environmental, economic and social level. It is the most comprehensive concept of justice at all levels. It is concerned with the improvement of the quality of life and the promotion of equal opportunities between developing and developed countries alike, while bringing about radical and societal changes with integration framework of political interest and legislation to ensure sustainability in all fields. The study also examined benefits for achieving architectural sustainability according to the studies carried out in the sustainable buildings, in addition to the principles of design which are three points (Economy of Resources - Sustainable Life Cycle for Buildings- Humane Design). The study also examined the analytical model of the building " Conference Center in Vancouver - Canada" the building represen practical application of sustainable building. Finally, the research also addresses the practical consequences of applying sustainability to the building.
\end{abstract}

KEY WORDS : Economic Sustainable, Environmental Sustainable, Community Sustainable Architecture Sustainability Principles, Sustainable Design Principles.

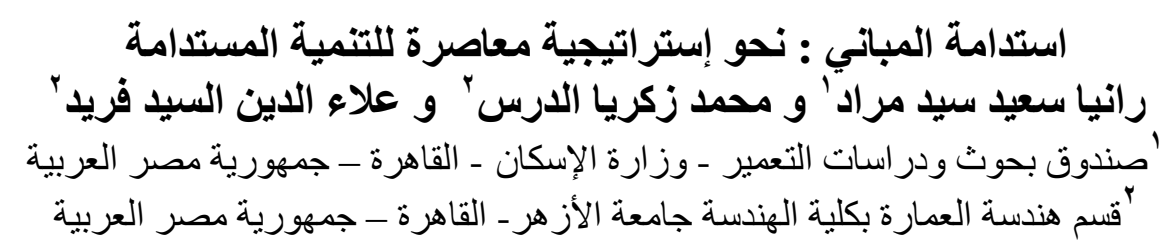

ملخص البحث

تناول البحث بالدراسة مفهوم الاستدامة على المستوى البيئي والإقتصادى والإجتماعى ، إذ إنها المفهوم الأ شمل للعدالة بكافة

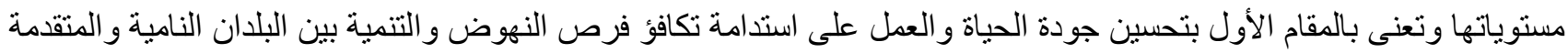

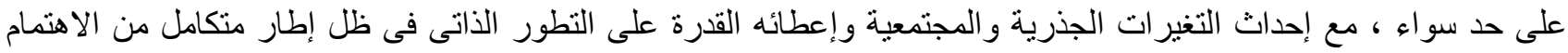

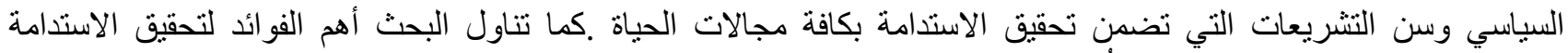

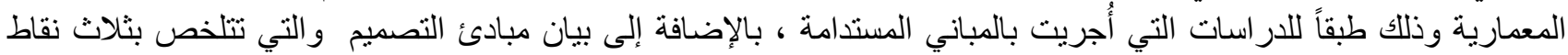

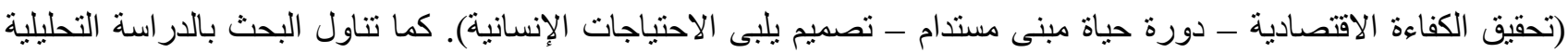

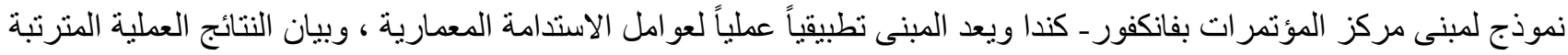

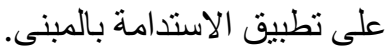

الكلمات المفتاحية : الاستدامة الاقتصادية ـ الاستدامة البيئية ـ الاستدامة المجتمعية ـ مبادئ الاستدامة المعمارية ـ مبادئ

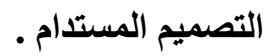

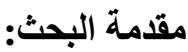

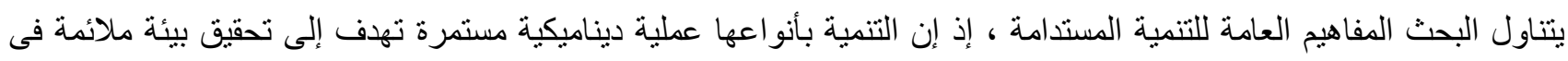

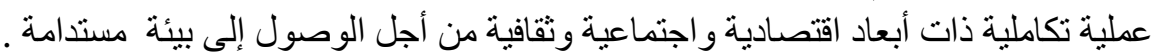


وسيتناول البحث دراسة للاستدامة المعمارية والفو ائد من تحقيقها ، وكيفية تجاوز الفهم التقليدي للاستدامة ؛ وذللك لخلق تناغم ما

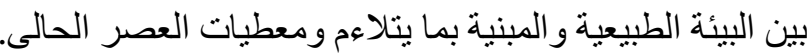

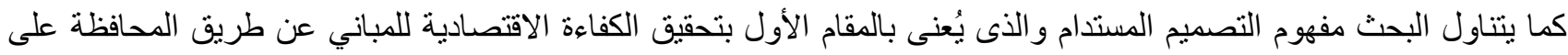

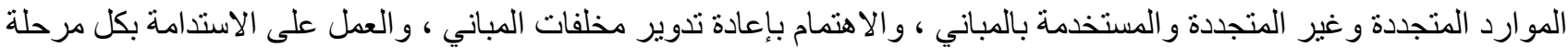

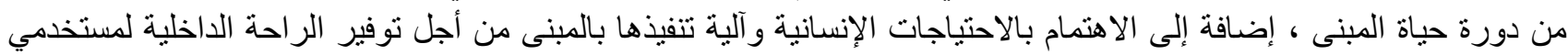

المبنى بكافة أنثكالها. ويتتاول البحث بالدر اسة التحليلية نموذج لمبنى مستدام ؛ لبيان النتائج العملية المترتبة على تطبيق الاستدامة به.

المشكلة البحثية:

تكمن المشكلة البحثية فى تز ايد المشاكل البيئية العالمية مثل الاحتباس الحر اري وزيادة معدل استهلاك الطاقة و غير ها من المشاكل

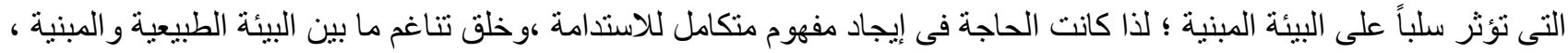

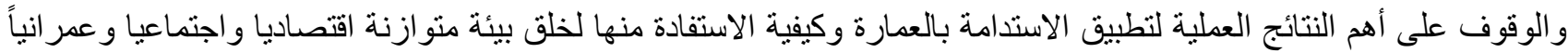

نحو آفاق ورؤى مستقبلية مستدامة.

الفرضية العلمية:

أن التصميم المستدام هو النقطة التى من خلالها تتحقق الاستدامة فى شتى مجالات الحياة ، فالوصول التى الاستدامة المعمارية

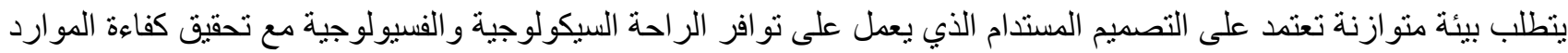

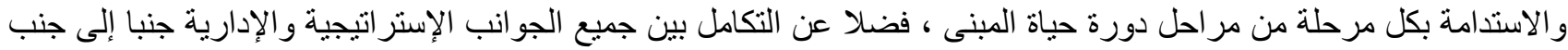

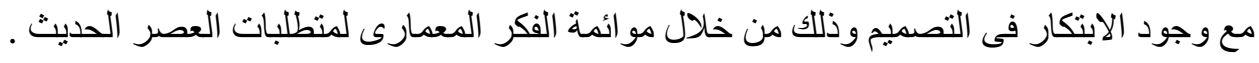

الهزف من البحث:

يهدف البحث إلي إيضاح مفهوم الاستدامة ، و الفو ائد المكتسبة من تحقيق الاستدامة العمر انية وكيفية الوصول إلى تصميم مستدام

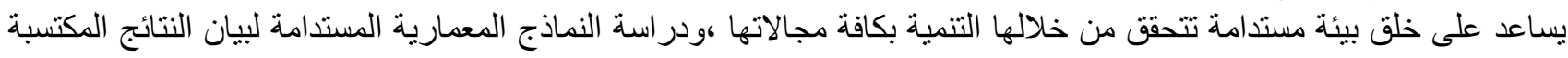
اقتصاديا واجتماعيا و عمر انيا من تحقيق الاستدامة المعمارية.

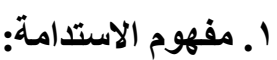
لتحقيق الاستدامة الثاملة يجب الاستة الاهتمام بآليات تطبيقها على المستوى الإقتصادى والإجتماعى و البيئي لضمان استدامة فعالة وفيما

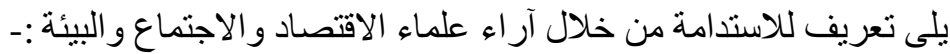

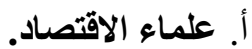

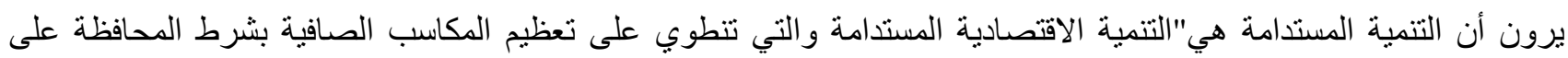

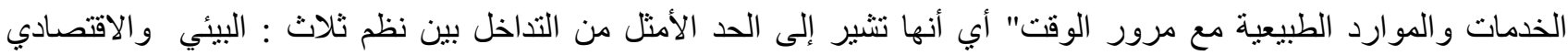

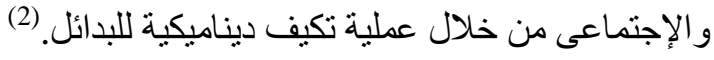

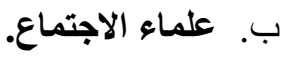

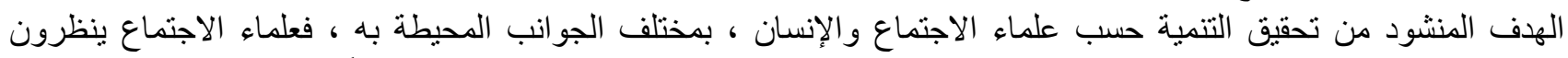

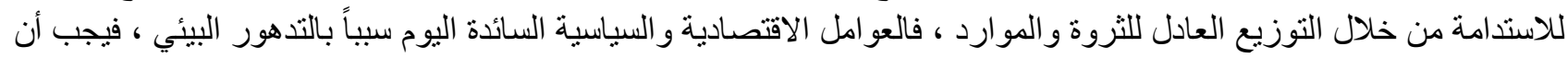

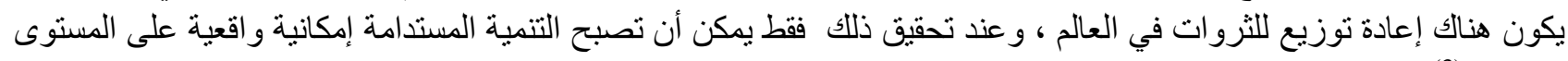
(3) العالمي.

\section{ج. عاء علماء البيئة.}

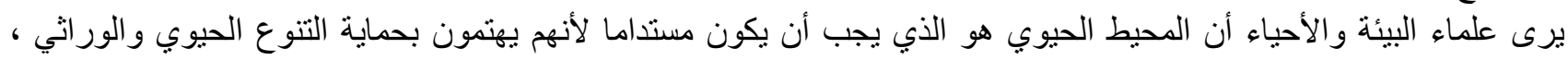

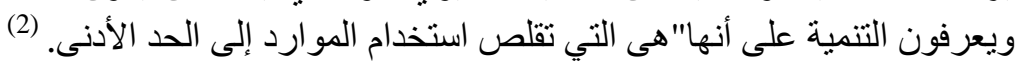

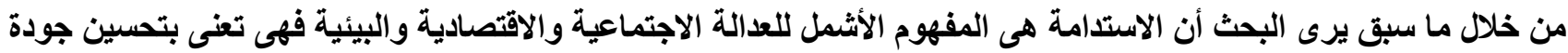

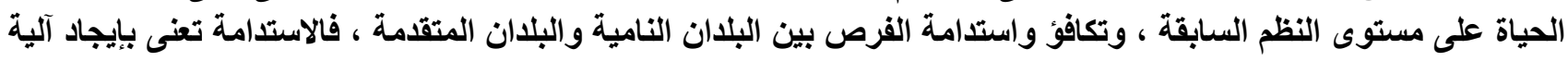

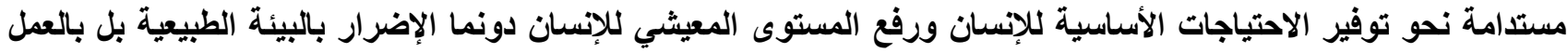

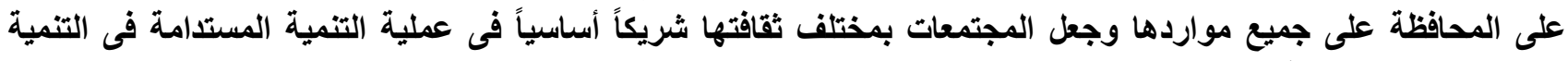

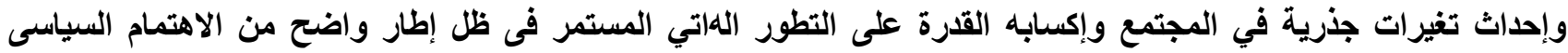
والقوانين والتشريعات التى تضمن استدامة بئية واتئ واقتصادية واجتماعية. 
r. التوافق المنظومى للتحقيق التنمية المستدامة:

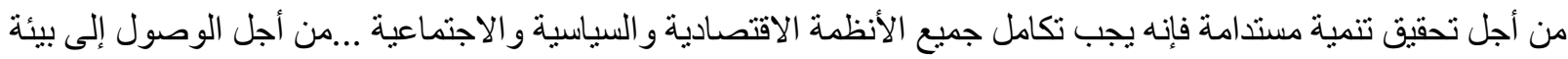

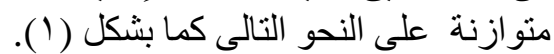

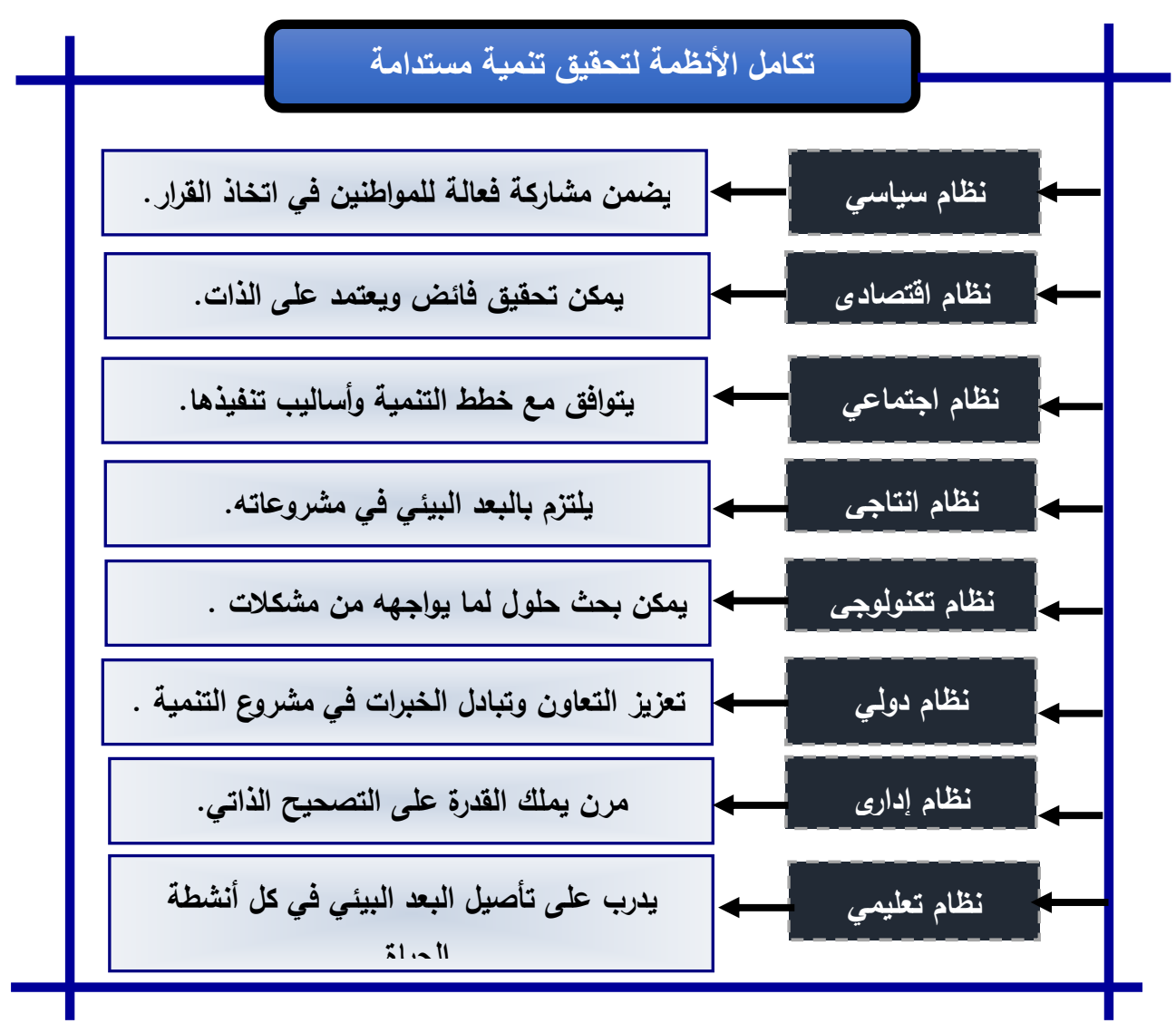

شكل (1) تكامل الانظمة لتحقيق التنمية المستدامة

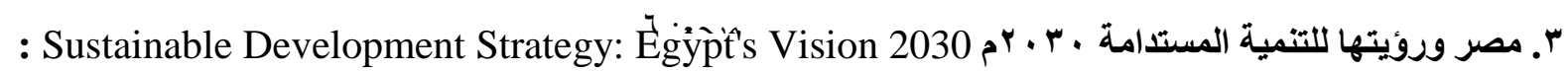

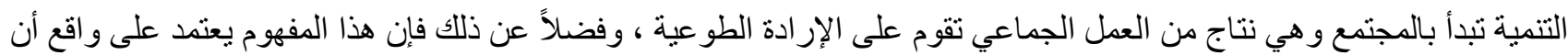

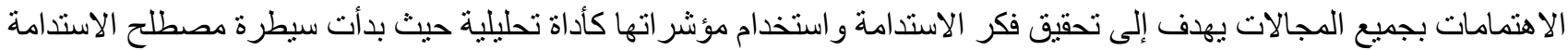

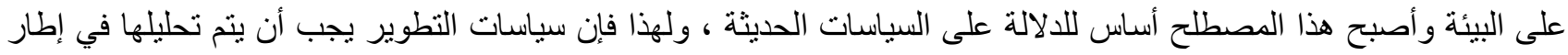

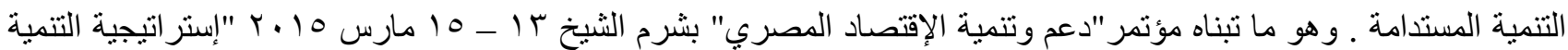

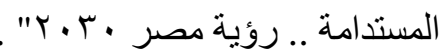

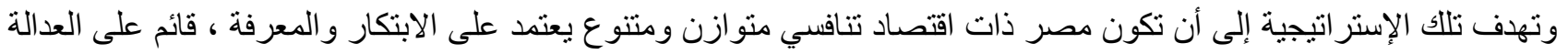

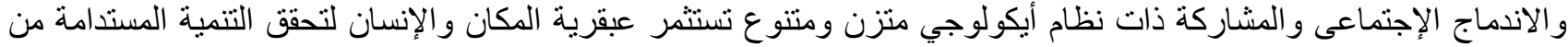

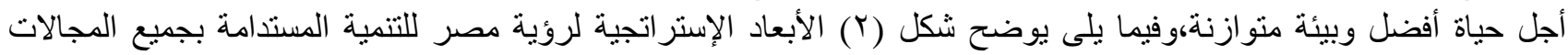

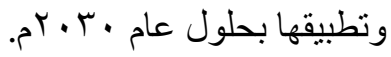




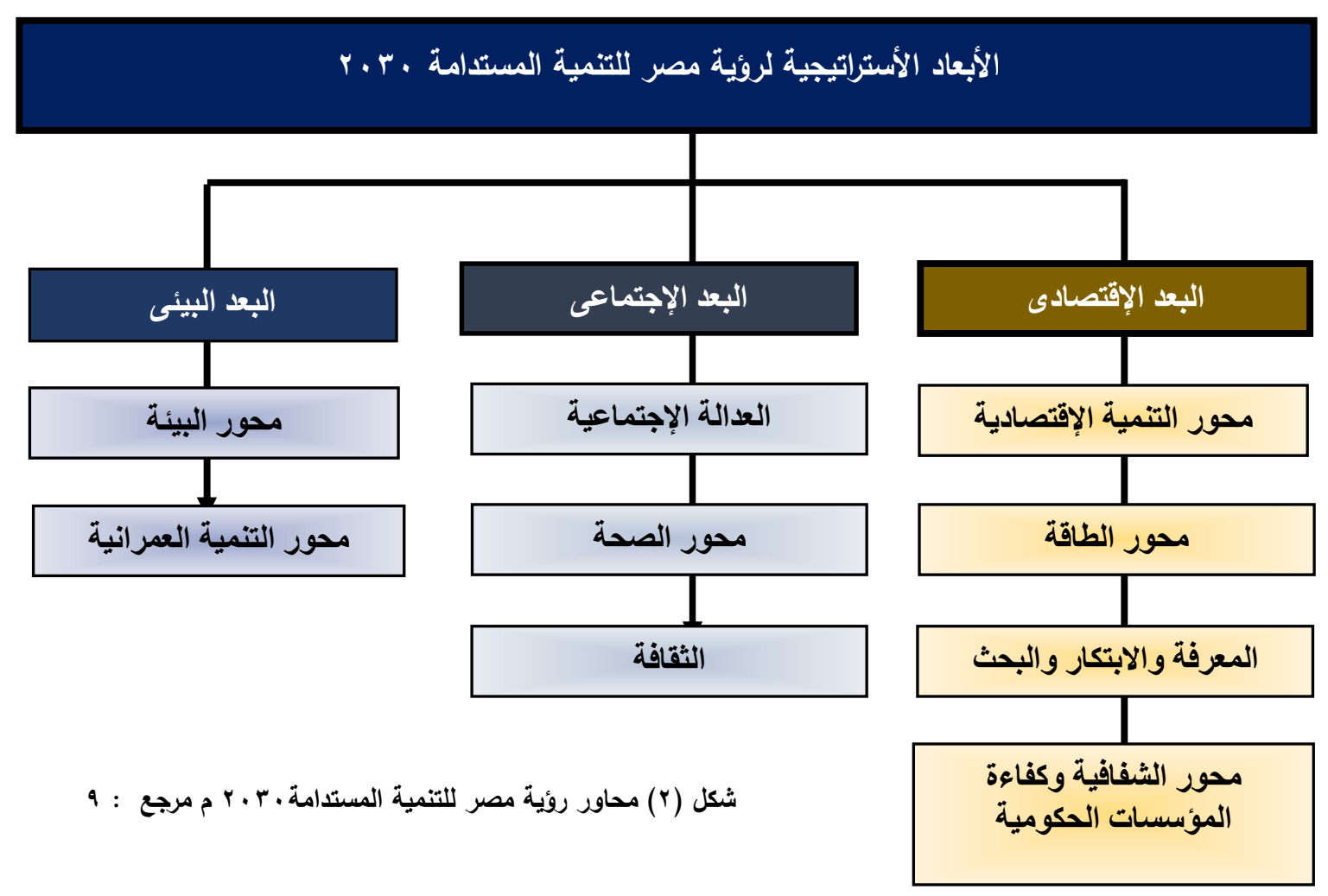

ويهتم البحث بدر اسة محور التنمية العمر انية وتحقيق الاستدامة به ،وفيما يلى جدول ( 1 ) عرض لأهم الآر اء التى ناقثت مبادئ الاستدامة العمر انية كما يلي:

؛. أهم الفوائد المباثرة لتحقيق الاستدامة العمر انية :

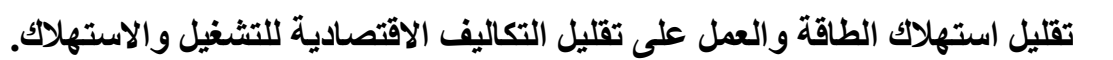

إنشاء مباني مستدامة ليست بالتكلفة العالية وان كانت التكاليف الأولية مرتفعة قليلاً بالمقارنة بالمباني التقليدية ، لكن ذللك يقابله انخفاض تكاليف التشغيل و الصيانة.

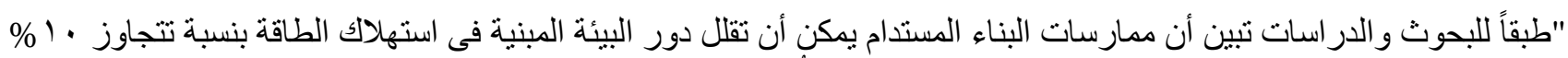

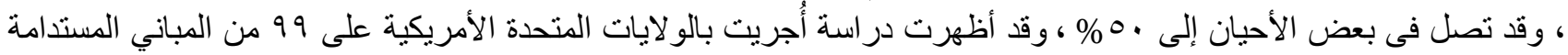

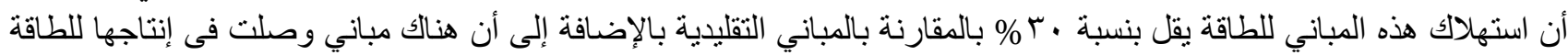

إلى •0\% من استهلاكها".

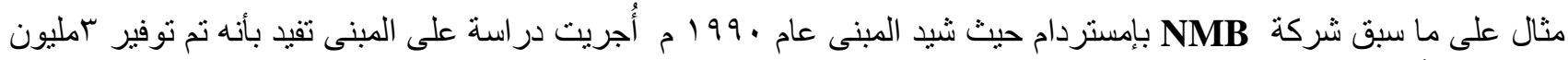

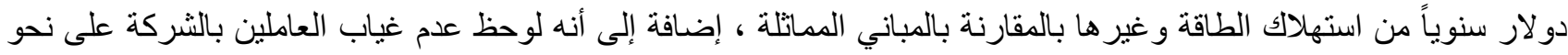

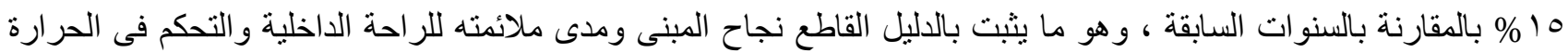

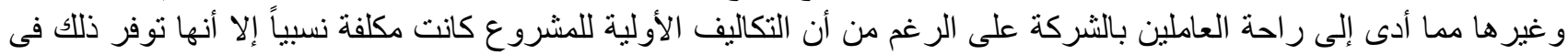

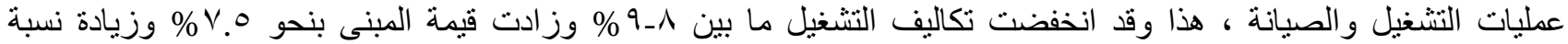

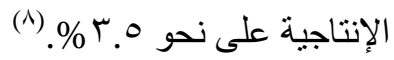

\section{زيادة القدرات الإتتاجية}

المبانى المستدامة لها آثار ها الاجتماعية على صحة وساجة وسلامة شاغلى تلإك المبانى ، وقد أدت مميز ات التصميم الذى يعزز الاستدامة

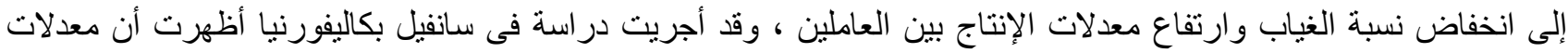

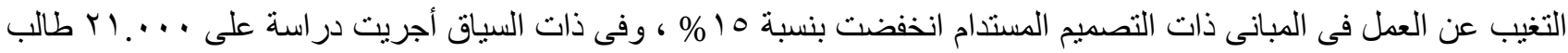

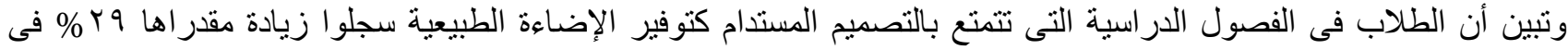

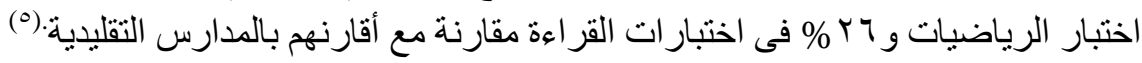




\begin{tabular}{|c|c|}
\hline مبادى ، الاستدامة العمر انية & اسم المؤلف (المصدر) \\
\hline $\begin{array}{l}\text { Economy } \\
\text { supporting communities } \\
\text { Creating Healthy Environment } \\
\text { Enhancing biodiversity } \\
\text { Minimizing pollution }\end{array}$ & $\begin{array}{l}\text { Halliday, S. Sustainable } \\
\text { Construction; Butterworth } \\
\text { Heinemann }\end{array}$ \\
\hline 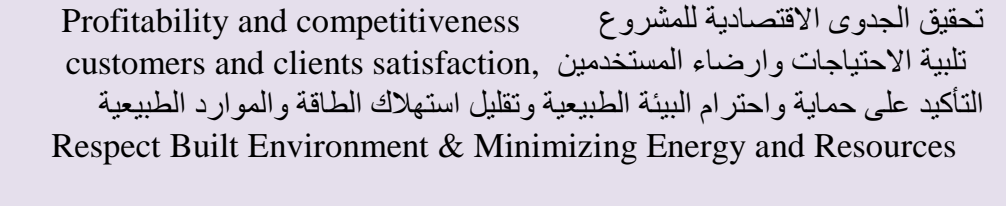 & $\begin{array}{l}\text { DET"R. "Building a } \\
\text { Better Quality of life: } \\
\text { Strategy for more } \\
\text { Sustainable Construction" }\end{array}$ \\
\hline 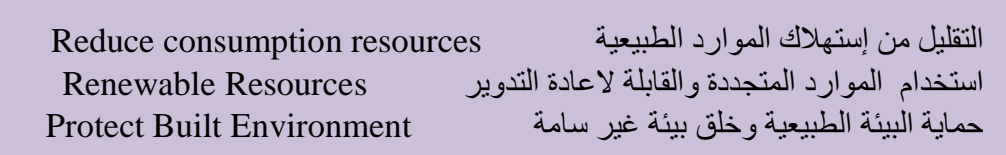 & $\begin{array}{l}\text { Miyatake, Y." Technology } \\
\text { development and } \\
\text { sustainable construction" }\end{array}$ \\
\hline 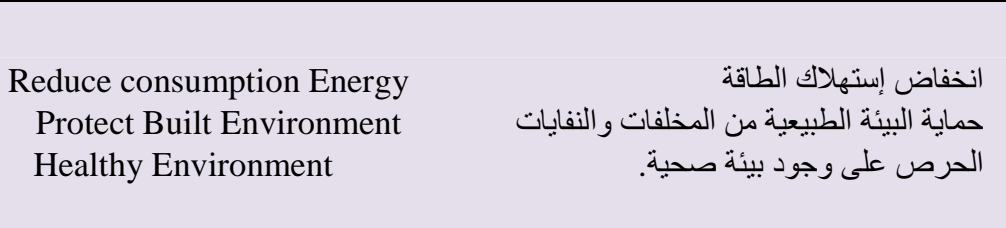 & $\begin{array}{l}\text {. Cole, R Coexisting } \\
\text { building environmental } \\
\text { assessment methods. } \\
\text { Building Research and } \\
\text { "Information }\end{array}$ \\
\hline 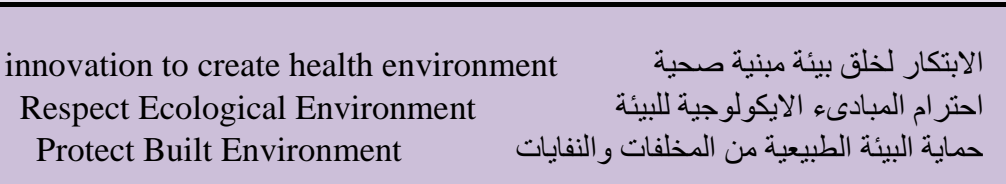 & $\begin{array}{l}\text { Kibert, C.J. "Sustainable .. } \\
\text { Construction: Green } \\
\text { Building Design and } \\
\text { Delivery" }\end{array}$ \\
\hline
\end{tabular}

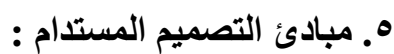

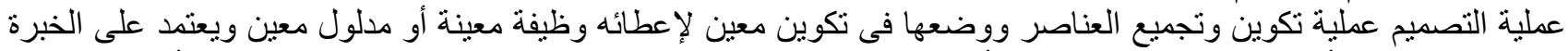

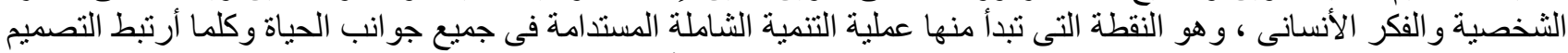

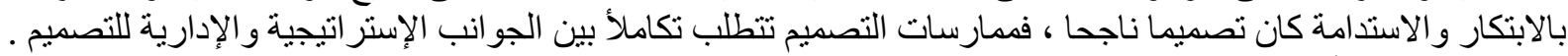

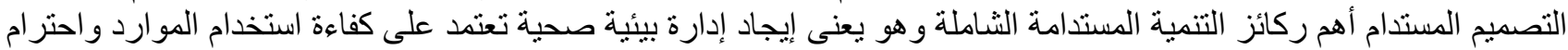

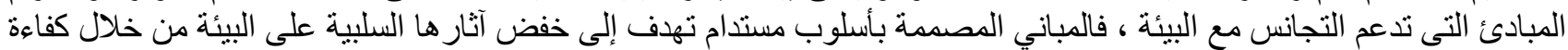

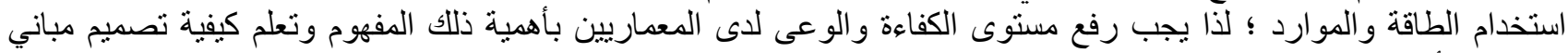

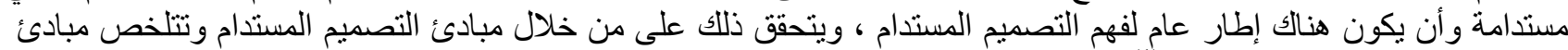

أ. أت تحقيق الكفاعة الاقتصادية فى الموارد المستخدمة للبناء.

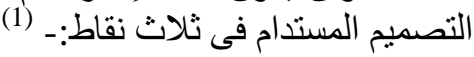

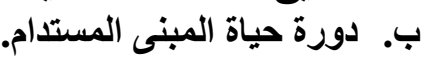
ت. تصميم يلبى الاحتياجات الإنسانية.

فيما يلى أحد الأمثلة العالمية كتطبيق عملى حول آلية تحقيق التصميم المستدام والوقوف حول أهم النتائج الاقتصادية والبيئة و الاجتماعية لتحقيق الاستدامة بالمباني :

7. مركز المؤتمرات بفانكوفر

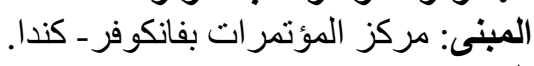

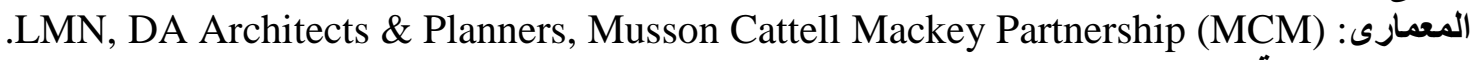
منست الإستدامة :Sustainability Coordinator: مuتمان المالك:BC Pavilion Corporation (PavCo) 
إستشارى البيئة : EBA Engineering Consultants Ltd.

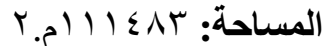

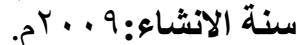

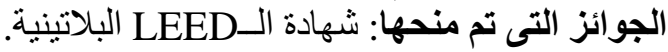

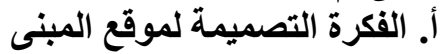

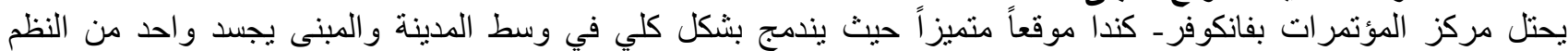

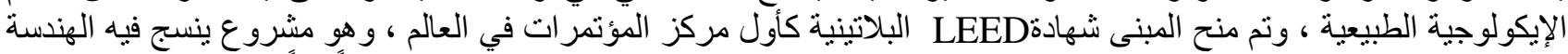

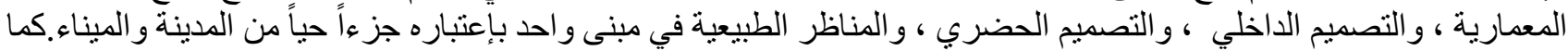

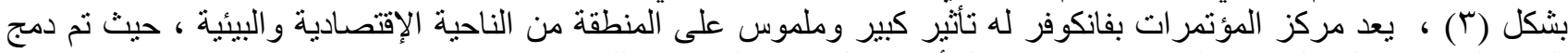

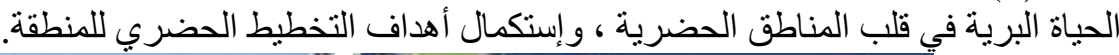
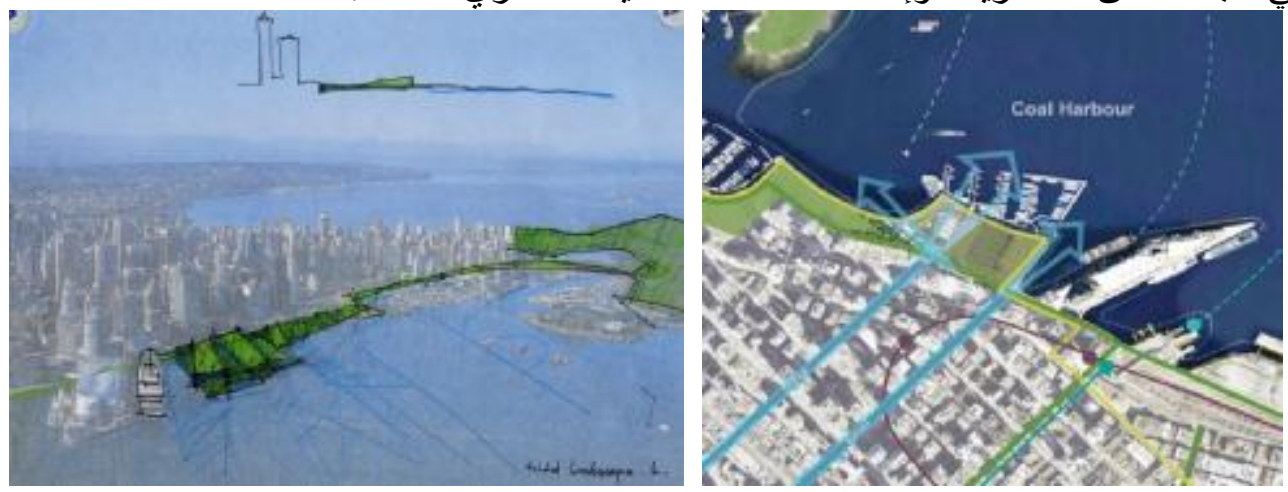

شكل(r) موقع المبنى الاستراتيجي بالنسبة لمدينة فارنكوفرا بكندا مرجع: .

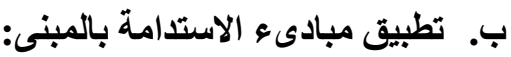

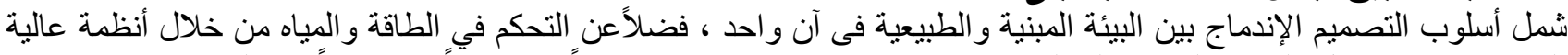

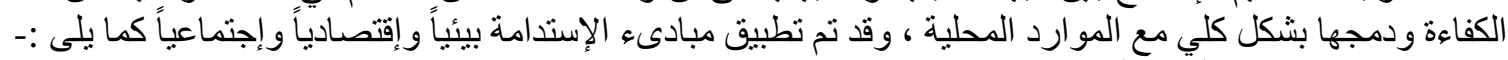
> الإستدامة البيئة:

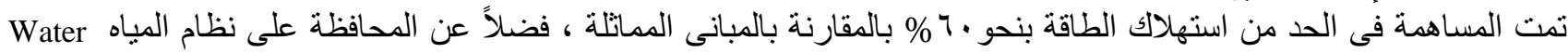
Conversion

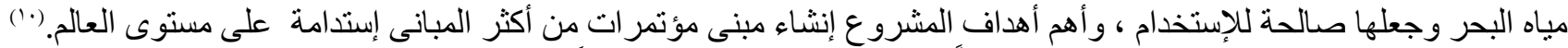

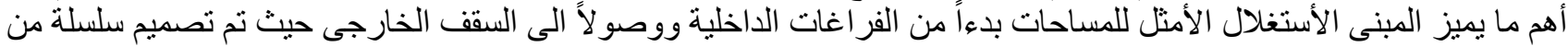

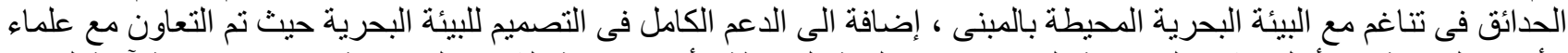

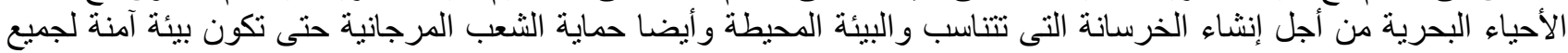

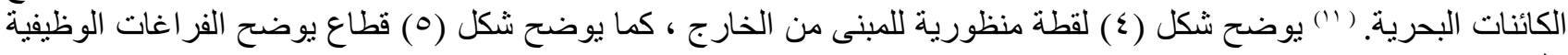

بالمبنى.

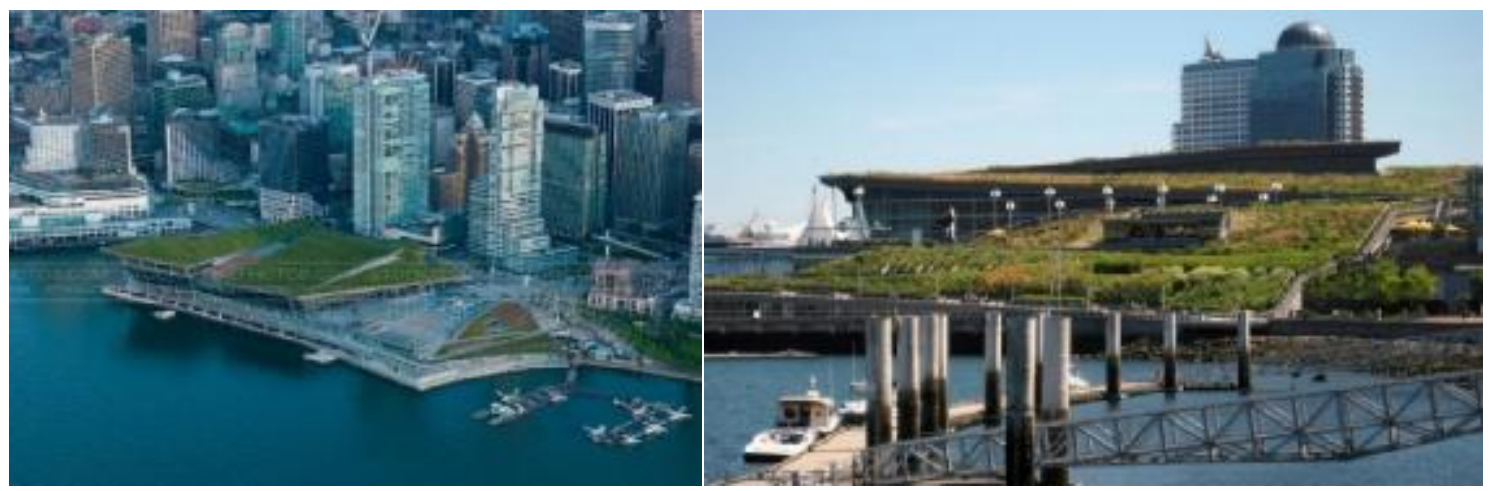

شكل ( ) لقطة منظورية لمبنى مركز المؤتمرات من الخارج المرجع : 11 


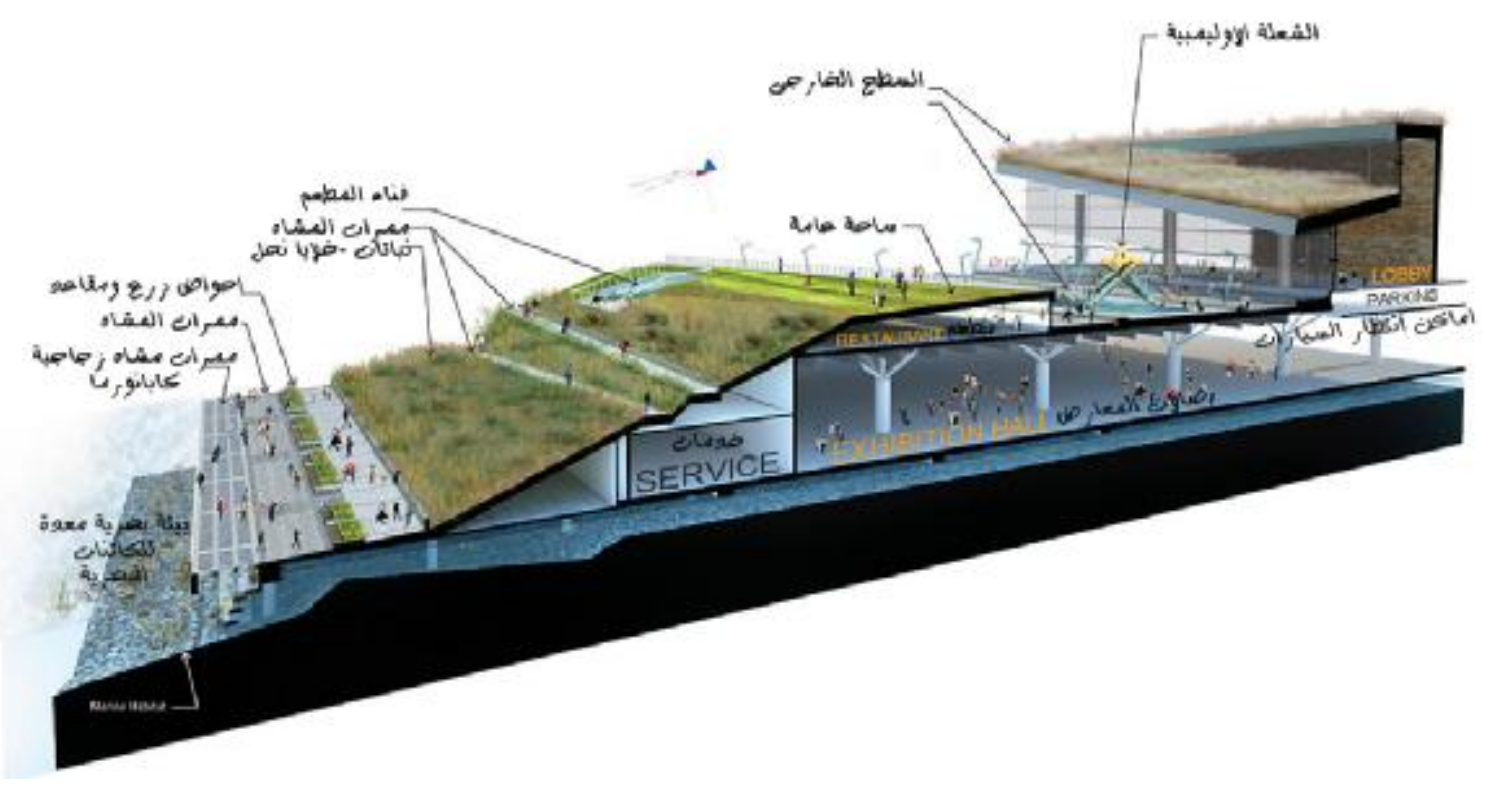

شكل (•) قطاع يوضح المعالم الرئيسية بالمبنى مرجع : ( )

أستغرق تصميم المبنى و إعداد رسوماته ما يقرب من ثلاث أعوام وكان العميل جزءاً أساسياً ومتكاملا مع فريق التصميم بالإضافة

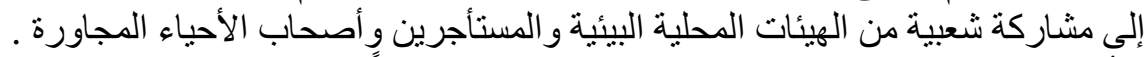

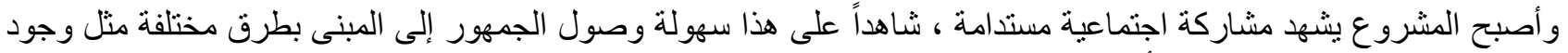

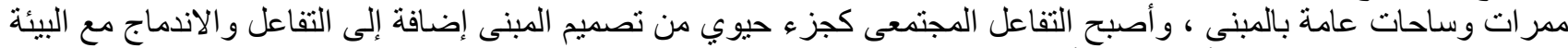
الطبيعية وبهذا تم تحقيق ركن أساسي من أركان الاستدامة للوصئ الإسول إلى عمارة مستدامة.

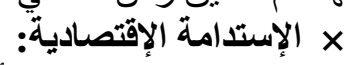

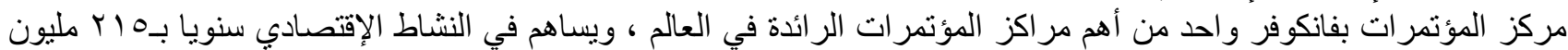

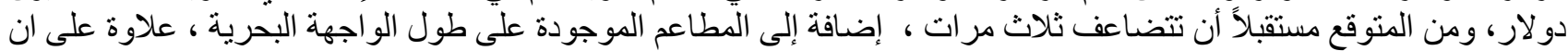

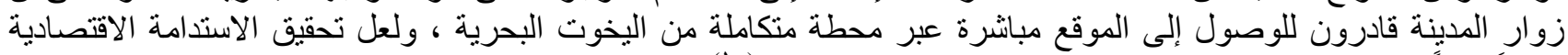

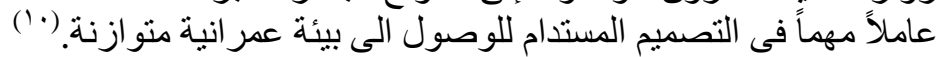

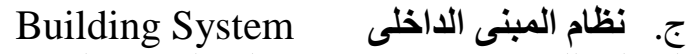

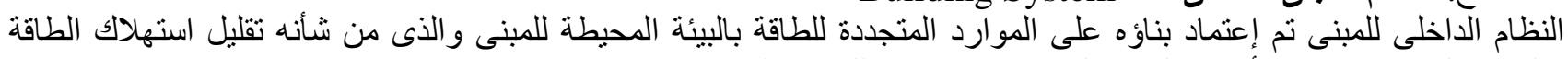

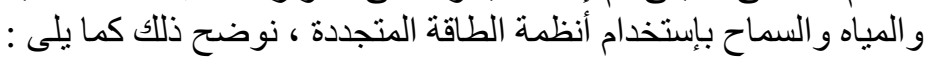

\section{Dergy Conversation أولاً: الحفاظ على الطاقة}

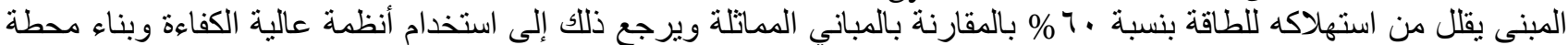

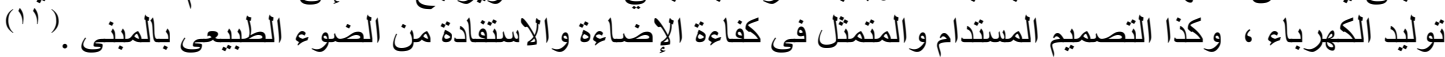

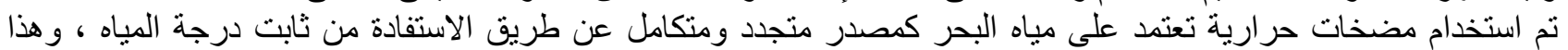

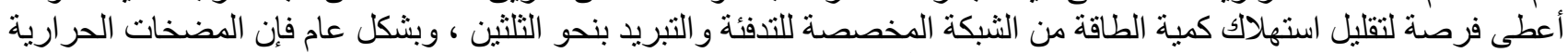

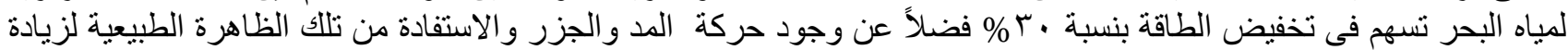

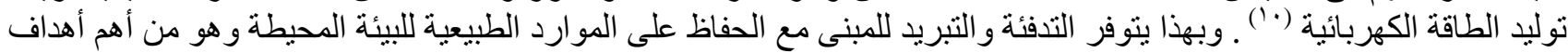

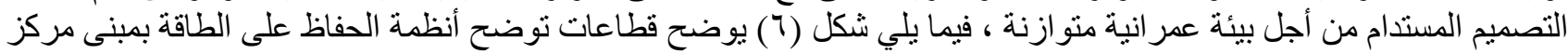
المؤتمر ات لمدينة فارنكوفر بكندا. 


\section{:Water Conversation ثانيا:الحفاظ على الموارد المائية}

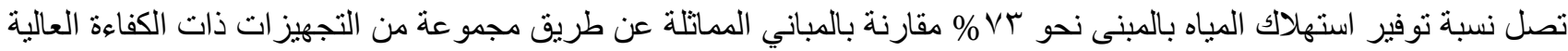

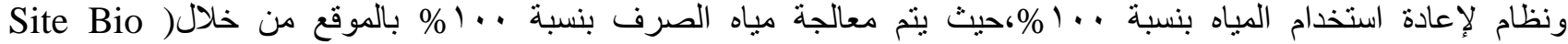

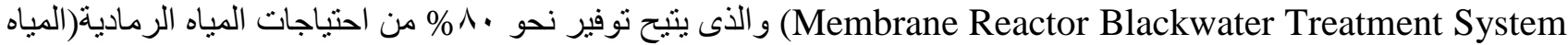

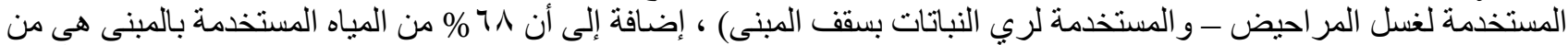

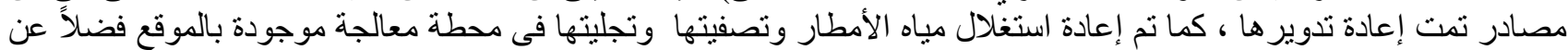

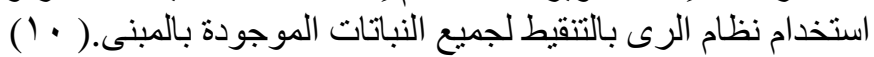

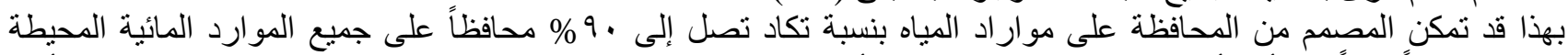

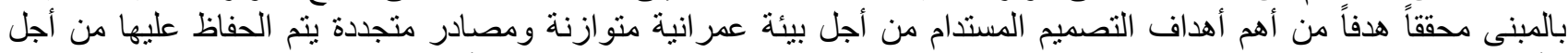

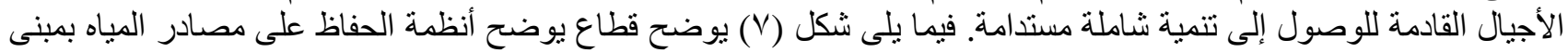
مركز المؤتمرات لمدينة فارنكوفر بكندا.

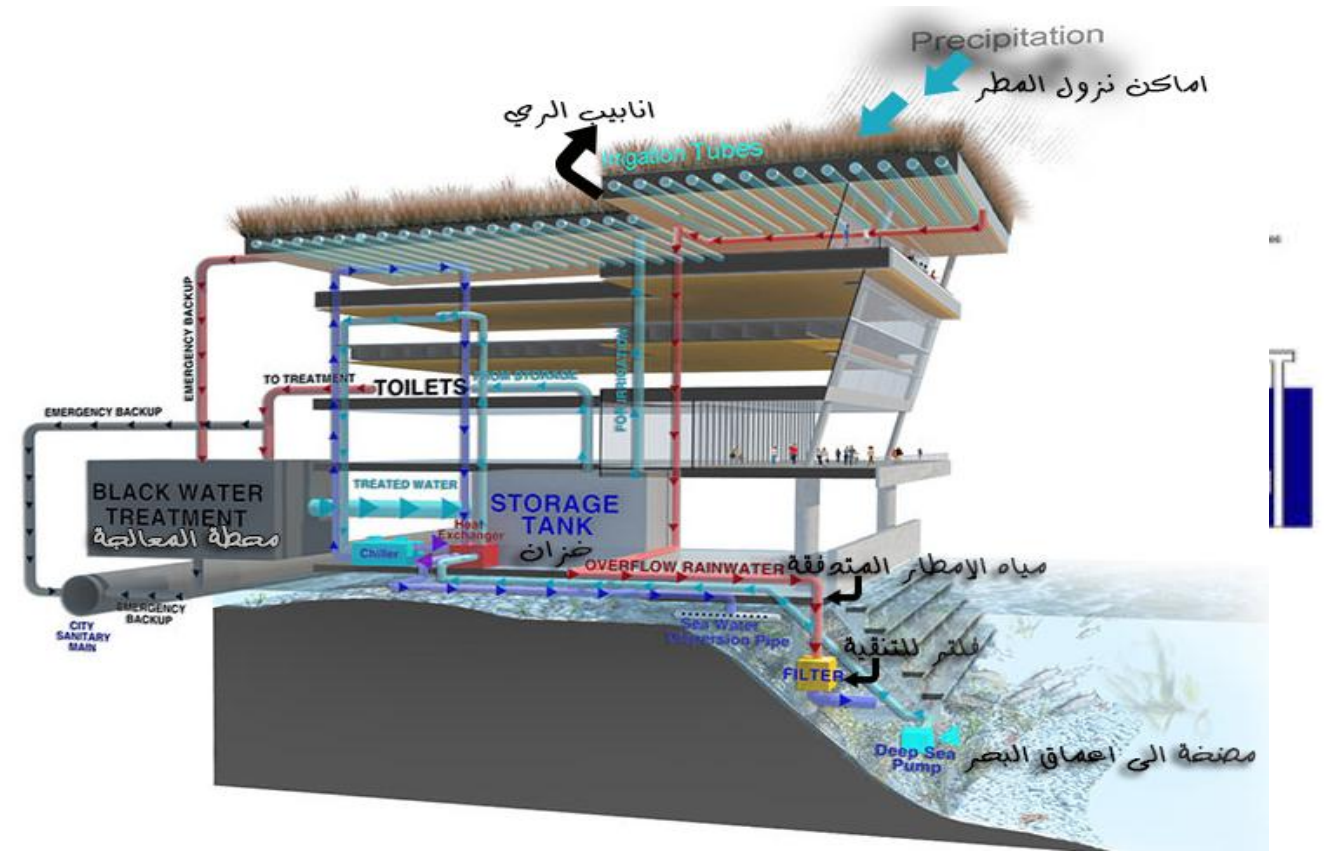

شكل (V) قطاع يوضح دورة المياه بالمبنى مرجع: 11

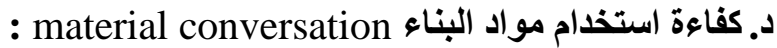

تم اختيار التشطيات الداخلية بعناية من مواد معاد معاد تدوير ها ، إضافة إلى قوة التحمل ، و واستخدام المواد المتجددة ، وكان التهان التركيز

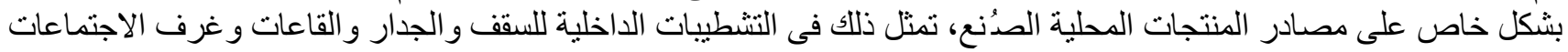

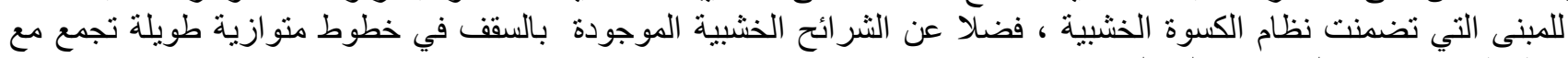
خطوط متعامدة من المساحات الداخلية. وقد تم تحقيق المعايير التالية نتيجة كفاءة مو اد البناءة البناء:

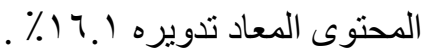

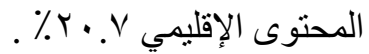

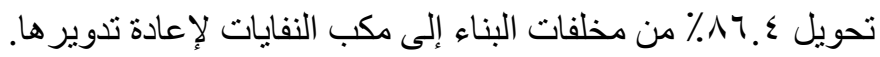

تم الحد من التعرض لثاغلي المبلى الثنى و عمال الصيانة للملوثات الكيميائية الخطرة التي تؤثر سلبا على نوعية الهو اء، و البيئة

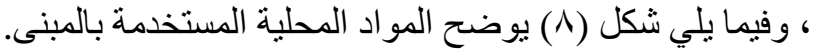




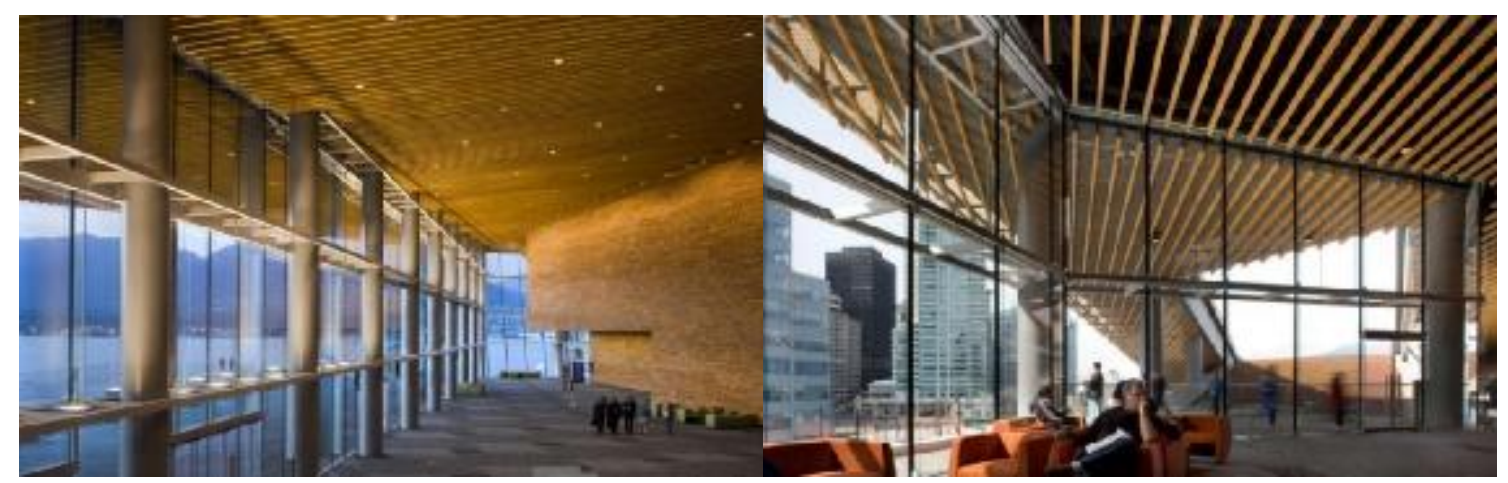

شكل (^) استخذام مواد البناء المحلية لمبنى مركز المؤتمرات مرجع : 11

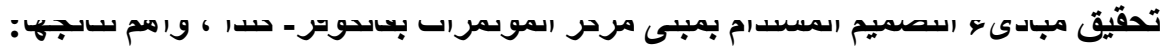
وفيما يلى جدول (r) يوضح أهم العوامل الإستدامة بالمبنى والنتائج المترتبة على ذلتك كما يلى :

جلول (r) عوامل الإستدامة بمبنى مركز المؤتمرات بفانكوفر ونتائجها المصدر: الباحث

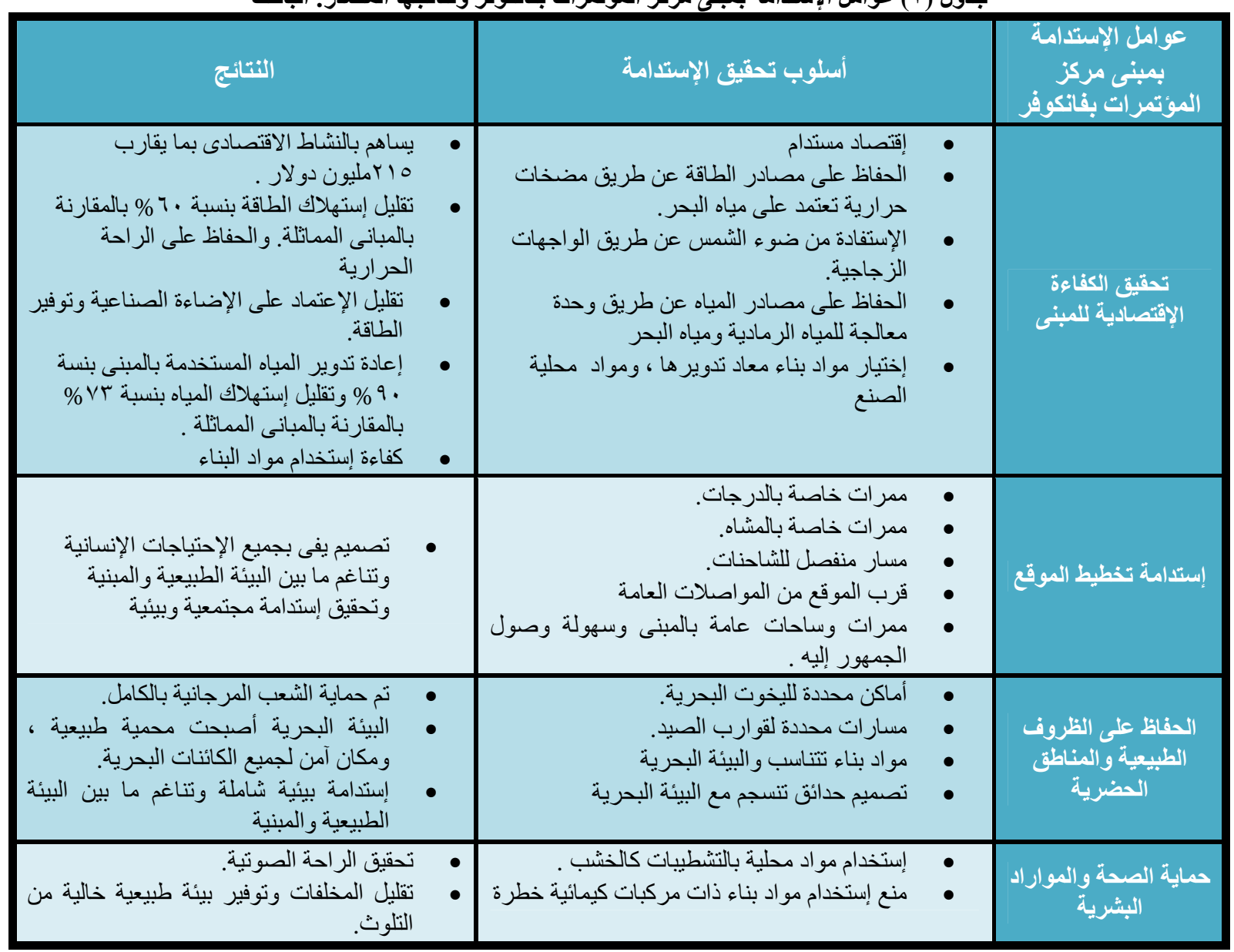

إن در اسة مثل هذا النموذج الناجح عالمياً بنطبيق الاستدامة بساهم بالوصول إلى النتائج الفعالة للاستدامة بيئياً ومجتمعياً و اقتصاديا

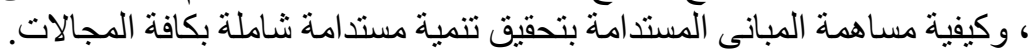


ا. ا. تكاملج الأنظمة المختلفة (السياسية ـ الاقتصادية ـ الاجتماعية ـ التكنولوجية ـ الإدارية ـالتعليمية) ركيزة أساسية نحو تحقيق

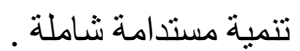

r. المباني المستدامة وسيلة مشتركة للتفاعل الإقتصادى والإجتماعى نحو إستراتيجية معاصرة لتحقيق استدامة فعالة بشتى المجالات.

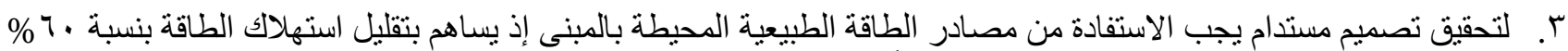

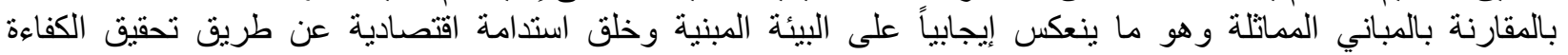
الاقتصادية للمبنى.

ع. للمساهمة فى خلق استدامة مجتمعية يجب مر اعاة استدامة تخطيط الموقع العام للمبنى و إيجاد العناصر التصميمية التى تخلق تناغم ما بين البيئة الطبيعية و المبنية.

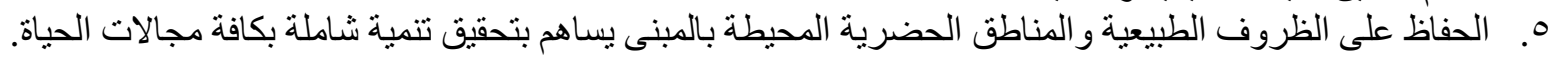

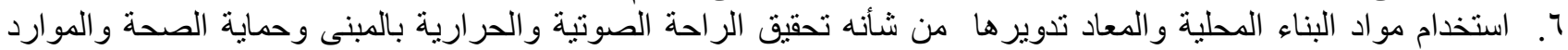
البشرية.

V. يجب على المعماريين والمخططين الآن، اختبار وتعديل التكنولوجيا المتطورة التى تساهم بتحقيق مبادئ التصميم المستدام

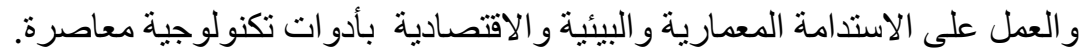

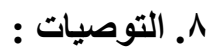

ا. العمل على نوعية الطلاب المعماريين بأهمية وطرق التصميم المستدام عن طريق تطوير المناهج والعمل على تطبيق مبادئ

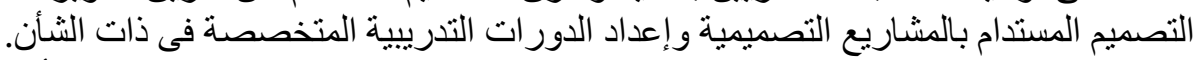

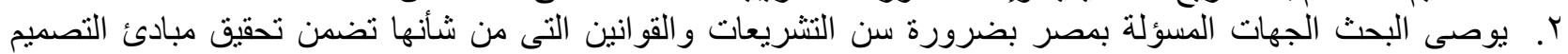

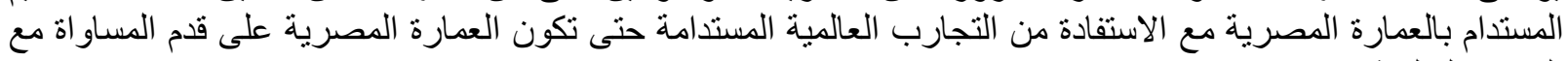

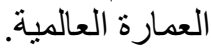
r. وضع إستراتيجية للتعاون مع الجامعات ومر اكز الأبحاث العالمية للتواصل العلمي للتعرف على أحدث الأبحاث المنشورة

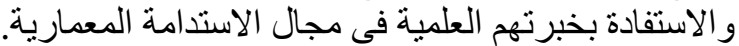
ع. تشجيع البحث العلمى نحو خلق أفكار تطبيقية للاستدامة واستخدام الأدوات التكنولوجية المعاصرة لخلق بيئة مستدامة عمر انياً وبيئياً و اقتصاديا.

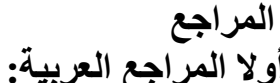

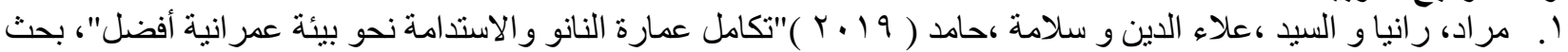

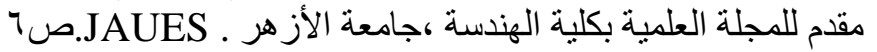

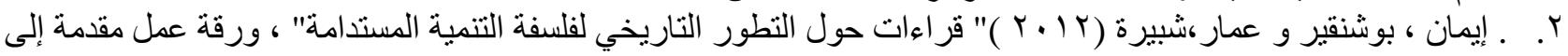

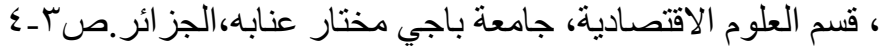

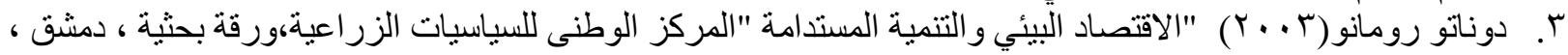

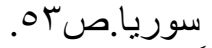

ثنانياً :المراجع الأجنبية

4. Kibert, C. J. (2016). Sustainable Construction: Green Building Design And Delivery. John Wiley $\&$ Sons.

5. Miller, W., \& Birkeland, J. (2011)" Green Energy. In Green Technologies: Concepts, Methodologies, Tools And Applications" (Pp. 1-16). Igi Global

6. Ellis, C. R. (2009). Who Pays For Green? The Economics Of Sustainable Buildings. Emea Research, 19.

7. Halliday, S. (2008)" Sustainable Construction" London: Routledge.

8. Council, U. G. B. (2003). Building Momentum: National Trends And Prospects For HighPerformance Green Buildings: Based On The April 2002 Green Building Roundtable And Prepared For The Us Senate Committee On Environment And Public Works. Us Green Building Council.

9. Http://Sdsegypt2030.Com ثنانياً :مواقع شبكة المعلومات الدولية

10. Vancouver Convention Centre West.” Https://Lmnarchitects.Com.

11. Vancouver Convention Centre West | Aia Top Ten Http://Www.Aiatopten.Org 